\title{
Virtual Scientific Models
}

\author{
Mieke Pfarr-Harfst \\ Department Information and Communication Technology in Architecture \\ Technische Universität Darmstadt \\ El-Lissitzky-Str. 1 \\ 64287 Darmstadt, Germany \\ pfarr@ika.tu-darmstadt.de
}

\begin{abstract}
Digital reconstructions are an important medium to open results of research to the public. The main potential of such reconstructions is that they give an opportunity to present the research in different ways. Besides this, during the last five years, digital reconstruction as a crossing point of various scientific disciplines, like architecture, history of architecture and archaeology, has become more and more a tool for researchers. By bringing the results of excavations into the third dimension they consolidate the knowledge of these disciplines. Serious questions or unclear situations of a building structure can be easier discussed and often be solved. This paper shows the potential of digital reconstructions as a tool for research at different stations of the research process and provides an insight into the research in this special part of the cultural heritage. The special potentials of this researching method are demonstrated on digital reconstruction projects done at the Technical University of Darmstadt over the last 20 years. Besides this, the paper takes a critical look into the future of the digital reconstruction as a researching tool, the chances and the risks of this method.
\end{abstract}

Researching tool. Cultural heritage. 3D-reconstruction.

\section{INTRODUCTION}

Digital three-dimensional reconstructions are used in the visualisation of cultural heritage for the transfer of knowledge as well as for research purpose models at the point where architecture, archaeology and the history of art and architecture meet. In the last five years these digital reconstructions have become increasingly a tool for science.

Through the paradigm shifts arising from digitality and, information and communication technology knowledge is available, within seconds, anywhere, independent of place and time.

Scientifically based digital reconstructions are a special form of the digital World Cultural Heritage and the New Memory.

After language and writing, we are entering the third generation for the passing on of the cultural memory of the world. (Koob 1995)

Within the process of scientific research the spectrum reaches from simple pictures in scientific papers, to the use of models coupled with a data bank or to an open research model. In the meantime, a wide variety of technical possibilities has become available for the practical implementation of such models. Thus, this paper will not concentrate on a technical system, but rather will be a theoretical view related to methodology, potentials and fundamental structures. Further the properties and the resulting potentials of virtual models are placed in the context of the process of scientific research in general and transferred to reconstruction projects.

\section{VIRTUAL SCIENTIFIC MODELS}

\subsection{Basic principles}

During the last decade most terms in use have become inadequate to express the underlying potential of digital reconstructions on a scientific basis. Rather, this type of scientific method should be called a Virtual Scientific Model. A Virtual Scientific Model is a digital three-dimensional model that can be employed in research in various ways.

Besides this, these Virtual Scientific Models are developed from long-standing working processes, which on the one hand deal with the computer implementation of the basis into threedimensionality, on the other with the continuous discussion among experts. These two processes take place in alternation. By means of this methodology, extant knowledge is fused into new three-dimensional data and new knowledge is 
generated. Bringing the results of research into the third dimension, they consolidate the knowledge of these disciplines. Serious questions or unclear situations of a building structure can thus be more easily discussed.

By means of digitality, three-dimensionality and the language of pictures as the basic properties and principles of such data it is possible to decipher complex constructional structures and processes over time and to pass on research results in a comprehensible manner.

So Virtual Scientific Models have two main property traits resulting from the principles outlined above. The first one is the merging and consolidation of knowledge and the second one the threedimensionality.

\subsection{Properties}

\subsubsection{Merging and consolidation of knowledge}

Bringing together all of the data relevant to a research project in an interdisciplinary working process is one of the most important characteristic traits of Virtual Scientific Models. Today most of the excavation data are collected digitally, and archives and libraries are also digitalizing older sources. In this context the virtual model can serve as an orientation system, a sort of interactive stage upon which the current state of information and research can be presented in a visual manner. All research data can first be merged and consolidated into such a model, which can be employed at the beginning, during and after the research or excavation process.

At the beginning such a model can show the status quo of a research project and serve as the basis for further research and/or excavation campaigns. During a research process a virtual scientific model can serve as an open research model. There is already a wide variety of technical possibilities, such as self-generating models, the coupling with data bank systems, and the use of caves. After the research project, the virtual scientific model can make knowledge widely available in a suitable documentation and archiving system.

Therefore the potentials of the merging and consolidation of knowledge are:

- Definition of the archaeological findings and sources - interactive stage

- Open research model

- Documentation und archiving

\subsubsection{Three-dimensionality}

The first potential of the characteristic trait threedimensionality is the illustration of complex relationships in space and time. The digital model becomes a tool for the scientific process in order to investigate open questions concerning a spatial structure in a content-based or time-based context, also for large architectural structures or areas. The use of model structures at varying scales, and thus with differentiated information densities, facilitates this process.

Working within three-dimensionality makes it possible to observe three-dimensional ideas, which are reconstruction variants, side-by-side. Older ideas can be reappraised, new ones can be established, contradictions exposed and new directions sought through interdisciplinary discourse. In relation to the research process, three-dimensional theses or sketches can be applied prior to beginning an excavation campaign or a research project in order to facilitate or complement the formulation of the scientific problem. During the research process, these threedimensional theses can be used as a guide for structuring and orientation. By means of concrete formulation of questions, these theses can be reviewed, refuted or verified. After the research process, the three-dimensional theses can serve by themselves as a basis for further research.

The transfer of constructions, assembly principles for construction elements and architectural styles into virtual three-dimensionality is quite comparable to building in reality, a further potential of threedimensionality. Within the scientific context, the transfer of sources into a 3D-model requires wider knowledge of the basis of architecture and construction as well as of the historic parameters.

Here one can simulate construction processes, environmental influences, cultural particularities and constructional-technical procedures. In particular, this potential can be applied during the research process and can support scientific experimentation.

The potentials of three-dimensionality are:

- Illustration of complex relationships in space and time

- Designing three-dimensional theses und variations

- Building in three-dimensions

- Understanding structures that are not visible

\subsection{Context: Culture Heritage}

In the transfer of knowledge, digital reconstructions as a visual medium have established themselves in the past 15 years. On the basis of the previously mentioned properties, these reconstructions demonstrate potentials that range much farther than visualisation. Exactly in this respect they will become future tools for the paradigm shift in the 
investigation of our cultural heritage. Given these properties, linked with the resulting potentials, there is a basis for further information for virtual scientific models.

\subsection{Potentials}

In the following the two properties are described coupled with the resulting potentials. During the past two decades many digital reconstructions of important historical buildings from around the world have been generated at the Department of Information and Communication Technology at the Technical University of Darmstadt. In addition to the classical uses for the transfer of knowledge, the focus was on the scientific application for threedimensional data as a scientific tool in each of these interdisciplinary and international research projects. Open scientific problems have been investigated and solved together with recognised experts directly at the computer during the process. On the basis of selected reference projects the potentials of digital reconstructions as virtual scientific models are identified and discussed; these potentials can fuse knowledge, generate new knowledge and thus become digital cultural heritage and a future tool for scientific investigation. These reference projects include the digital reconstruction of the architectural history of the Kremlin in Moscow, the Imperial Graves of Xian, the destroyed synagogues in Germany as well as of Byzantine Ephesus.

\subsubsection{The resulting potentials of the Property "Merging and consolidation of knowledge"}

\subsubsection{Definition of the archaeological findings and sources - interactive stage}

In the Ephesus model it was the intention to bring together the findings of 100 years of excavation work, a classic case of the merging of knowledge. First of all three large-scale structural models (macrostructure I) were designed: a Greek, a Roman and a Byzantine model. Each was then referenced according to a city district (macro II) and individual building (microstructure I). Individual objects that can then be found in the buildings were allocated to the Microlevel II.

An incredible amount of findings was available for each building, district and objects. The sources were digitalized in the event that they had not yet been available in digital form. Moreover, 3D-models or 3D-scan data of objects and building elements that had been prepared as a basis for modelling by other scientists were also worked into the model. Thus a meshwork of data was created that demonstrates the complexity of the excavations at Ephesus and emphasizes the potential for knowledge consolidation.
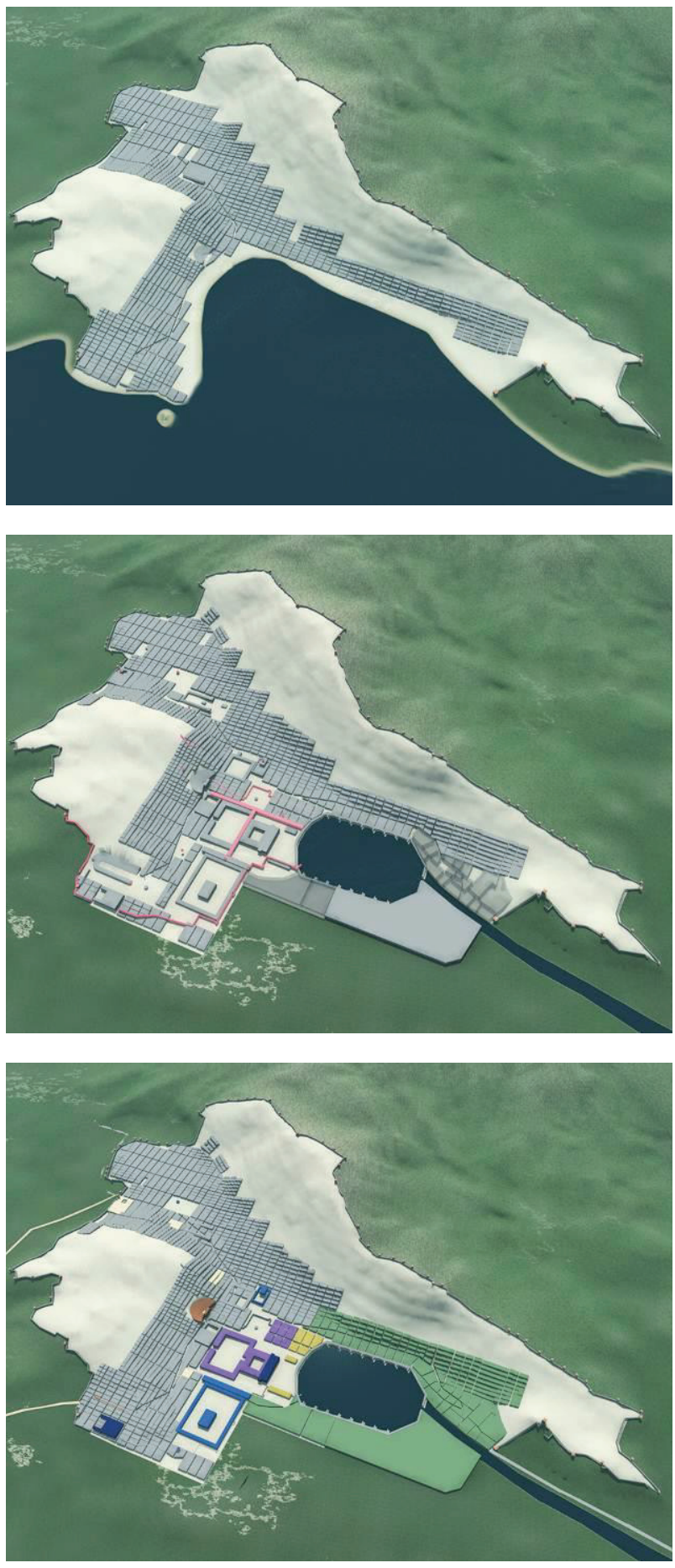

Figure 1: Ephesus in three periods, (c) TU Darmstadt

\subsubsection{Open Research Model}

The 3D-model of Ephesus became a sort of open research model that, since the completion of the original model, is undergoing a few extensions.

One extension included new findings concerning mill wheels and the masonry saw. This area was extensively revised.

\subsubsection{Documentation, publication and the availability of knowledge}

Within the context of digital cultural heritage and the new memory, all projects must include 
extensive documentation. The preservation of knowledge contained therein must be a goal of each project. At the Technical University of Darmstadt a special documentation system for digital reconstructions was developed for the retention of knowledge. The documentation system encompasses all components of digital reconstruction and is based on the basic principles of scientific documentation, of the London Charter and of known ontologies.

So the Four-Level-System arises as documentation structure:

- Level 1 comprises the background with general information and parameters for the reconstruction project,

- Level 2 the content of the project from the cultural, historical and architectural point of view.

- Level 3 involves methodology, the specification of individual provisions such as nomenclature, classifications, structuring must be coordinated with the question at hand.

- Level 4 is the level of objective evidence that constitutes the focal point and that fulfils the demand for scientific documentation, first of all the clear correlation of object and document.

\subsubsection{The resulting potentials of the Property "three-dimensionality"}

\subsubsection{3D Theses, variants or 3D-Sketches}

The starting point of the EU research project "Benedictine Monastery Plan" was to understand the plan of the St. Gallen monastery as a master plan which included construction instructions with the necessary information for building and space organization. "If one understands the monastery plan as a master plan, it must be applicable as a construction plan in northern, middle and southern Europe." In this case three 3D theses were set up with respect to the transfer of monastery design into one typical of each of the three regions together with the experts. The characteristic features of the regions were taken into consideration with respect to building construction, materials and design.

With the reconstruction of the Imperial Tombs of Xi'an two problems were developed as threedimensional theses, on the one hand, the threedimensional thesis of the South Palace of the burial site Zhaoling and on the other, the thesis with regard to the entrance to the burial site. The tomb of the first Tang-Emperor and its reconstruction consists of a northern section, a southern gate and a southern palace and the actual burial site within the mountain. The excavations of the southern palace were just beginning when the reconstruction was in its inception.
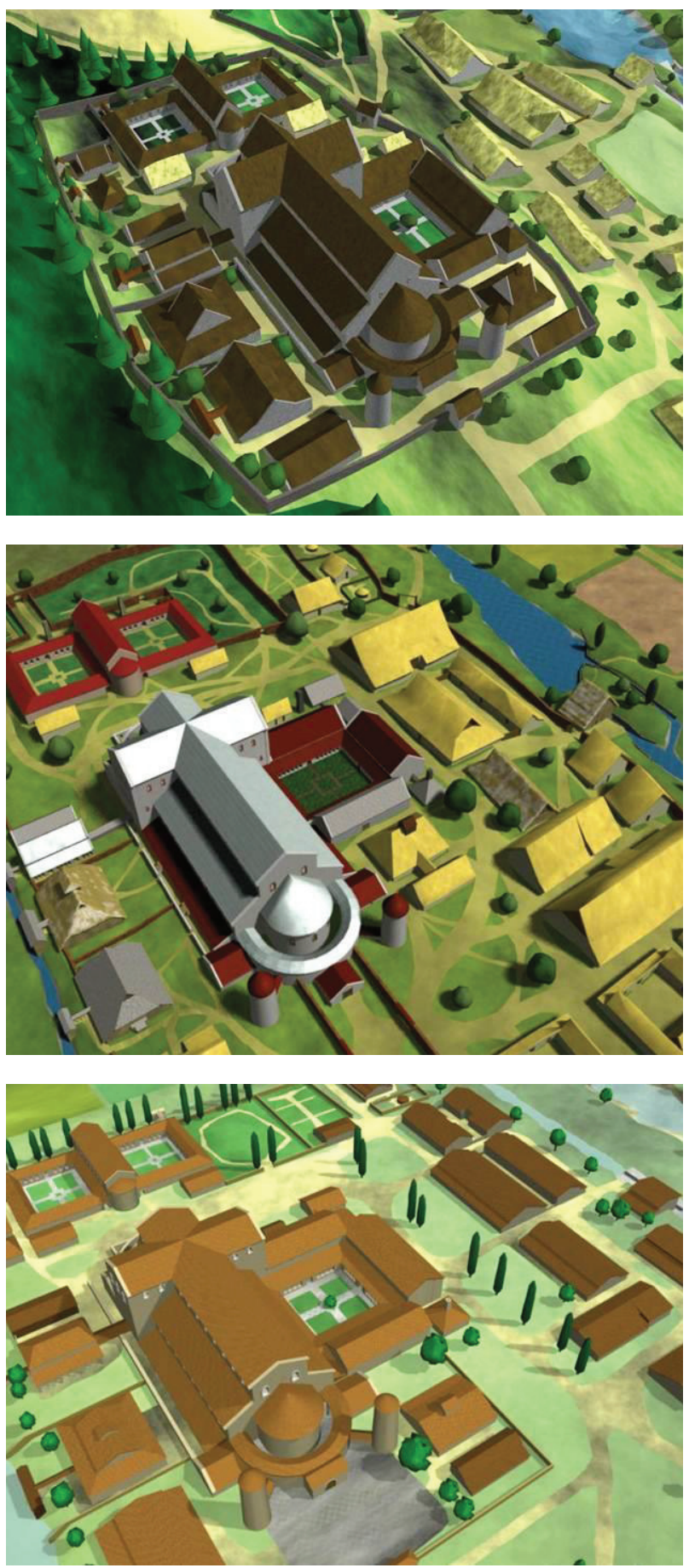

Figure 2: Benedictine Monastery Plan: North-, Middle-, South-Europe, (c) TU Darmstadt

The reconstruction is based on a few excavation findings and on geomagnetic investigations. The basic structure of the site was altered several times during the research process. The final result now serves as a basis for further research by the archaeologist. The entrance to the burial chamber had not been found at the beginning of the reconstruction work. There was only a single written source from a grave robber and an 
excavation finding with respect to a wooden bridge. The direct transfer of the written source, the locating of the sites of discovery as well as the comparison with other buildings led to a threedimensional thesis for a possible location of the entrance that, up to then, had been assumed to be at another location. After an on-site inspection, Prof. Zhang verified the presumed site of the entrance.

\subsubsection{Building in three-dimensions}

Construction in three-dimensional space as a direct transfer of active building in reality can be illustrated very well with the examples of the reconstruction of the Crystal Palace. The working plans for all of the building components were available for this "space without limits". Up to the present it has not been possible to reassess the assembly methods of this modular construction. As yet, it has not been possible to verify the construction methods of this ingenious feat of engineering. The transfer of each individual type of element into three-dimensionality and step-by-step assembly not only made the impression of space visible, but also this incredible engineering achievement is important for today's method of high-rise construction.
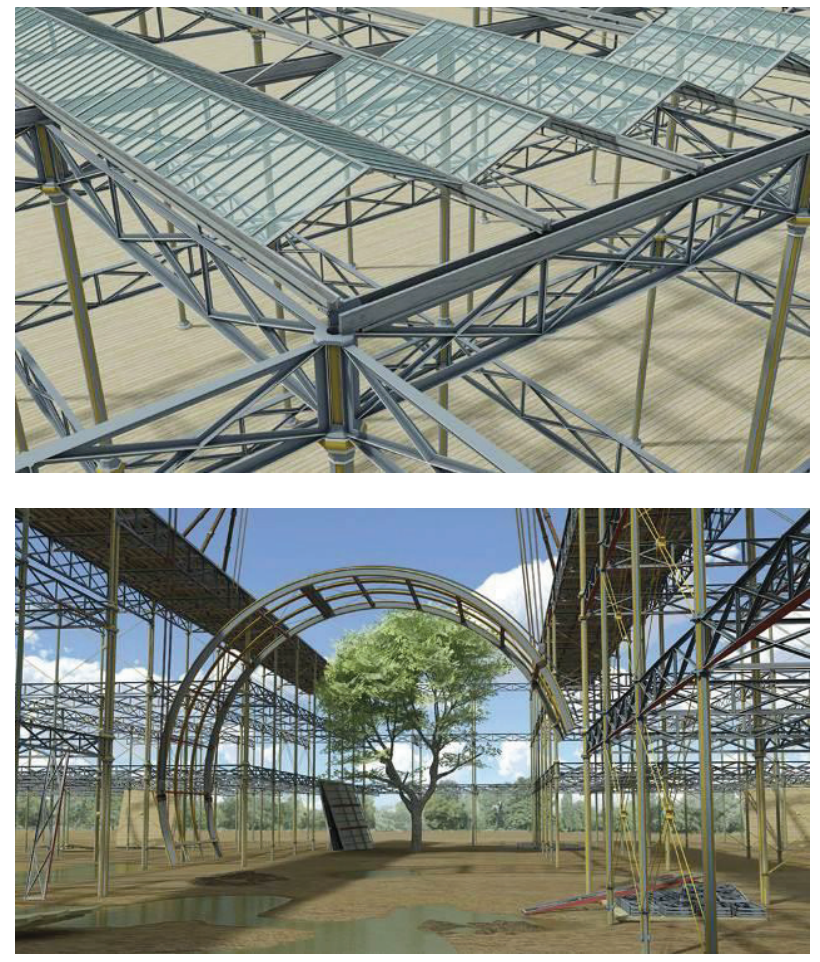

Figure 3: Crystal Palace, London, () TU Darmstadt

\subsubsection{Illustration of complex relationships in space and time}

To illustrate the potential of complex relationships in space and time two example projects are used.

The project "The Reconstruction of the Design and Construction History of St. Peter's Basilica in
Rome" as an example for the time components shows the layers of time in the context of the history and theory of architecture. A threedimensional representation of the 500-year history of the construction of the building was realized in the computer.

Each individual concept - model and sources - was provided with a suitable glossary and registered with its own data set. The referencing of the data did not take place until the end of the process. Thus the construction phases or plans of the architects and master builders can be superimposed as a whole by the data and an overview of the complex history of construction and spatial development is possible.

The excavation of the Cemetery of the Seven Sleepers in Ephesus can be utilized as an example of complex spatial structures. The Cemetery of the Seven Sleepers was a part of the general project Ephesus and thus incorporated in the data structure described above. Initially, all of the section and the outline were loaded. Together with the archaeologist systematically the entire complex was transformed three-dimensionally according to a certain system. First of all the basic structure and the external walls as well as the ceilings and floors were brought into the third dimension. After that the separating elements and the construction elements were gradually added. This systematic approach helped in the understanding of the complexity of three-dimensionality. This 3D-model showed that the old interpretations of this complex are not logical at all, new questions are posed. At the moment the 3D-model is only a three-dimensional thesis too. In the next step the archaeologist will try to answer these new questions inside the virtual scientific model.

\subsubsection{Understanding non-visible structures}

At last the potential "Invisible Structures" is described using the current project "Virtual Mathildenhöhe" as an example. The all-round work of art, the Mathildenhöhe, in Darmstadt, a center of Art Nouveau, was decisively influenced by the architect Josef Maria Olbrich. In a series of four exhibition phases he displayed his concept and that of other participating artists. The four exhibition phases showed different focal points. Today, however, not very much is discernible or tangible. The project will deal with all four exhibition phases and the attendant individual buildings. At the beginning of the project all sources like plans and photographs are loaded into the special data structures mentioned above. 


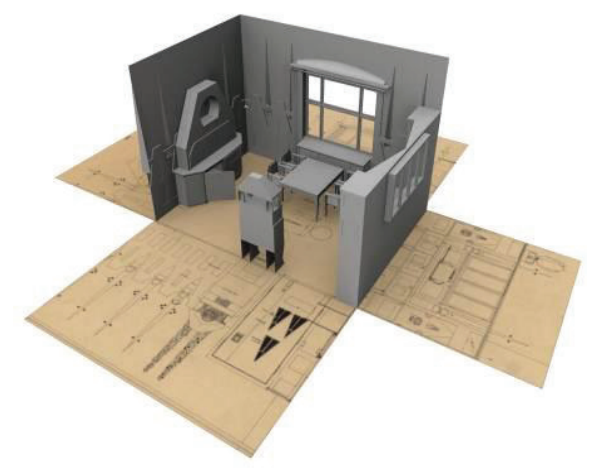

Figure 4: Mathildenhöhe Darmstadt - Dining room (House of Olbrich), (c) TU Darmstadt

The four exhibition phases are to be found at the macrostructure level II, which in turn is divisible into individual sectors - macrostructure level II. Individual houses, such as the Olbrich House, which was unfortunately destroyed, are part of the microstructure level I. The microstructure level II, rooms in the buildings, and the microstructure level III with objects complete the structure. The first goal of this project is to make the non-visible structures of the original complex "Mathildenhöhe Darmstadt" visible. Furthermore the second goal is to generate a prototype for an open, virtual scientific model as a basis for further research, in which existing technologies in this area will be examined and incorporated. The intention is to contribute to the preservation of digital cultural heritage and the new intangible memory and the general availability of knowledge. The bridge to the communication of knowledge is also built.

\subsection{Future challenge and vision}

The theoretical overview of the principles, properties, methodology and potentials combined with examples of real research projects showed the immense potentials of the Virtual Scientific Models as a tool for research. But at the moment in this part we are focused on the technical system, not on the basics like common principles.

In addition, especially in view of the preservation of digital cultural history and the still prevailing lack of standards, the present need for action and future challenge becomes clear.

Therefore a great research project is planned, bringing institutions and experts together to find a new way for the further research with virtual scientific models. The intention is to contribute to the preservation of digital cultural heritage and the new intangible memory and the general availability of knowledge. Perhaps that will be a new vision for research in digital cultural heritage.

In the long run there are future prospects for a vision of a scientific network, which intercommunicates by means of a virtual scientific model and thus generates new findings.

\section{REFERENCES}

Denar, H. (2009). Die London Charta. Für computergestützte Visualisierung von kulturellem Erbe. http://www.londoncharter.org/ (12.07.2009).

Frings, M. (2001) Der Modelle Tugend. CAD und die neuen Räume der Kunstgeschichte. Verlag und Datenbank für Geisteswissenschaften, Weimar.

Grellert, M. (2007) Immaterielle Zeugnisse. Synagogen in Deutschland. Potentiale digitaler Technologien für das Erinnern zerstörter Architektur. transcript Verlag, Bielefeld.

Guidi, G. (2008) 3D Modeling of Large and Complex Site Using Multi sensor Integration and Mulit-resolution Data. Eurographics Digital Library. http://diglib.eg.org/ (25.11.2012).

Heinel, K. (2011) Modellieren, Strukturieren, Präsentieren. Von Handaufmass bis High Tech III. 3D in der historischen Bauforschung. Verlag Philipp von Zabern, Mainz.

Hermon, S. (2007) 3D Modelling as a Scientific Research Tool in Archaeology. Academia.edu. http://www.academia.edu/1226903/3D_Modelling_ as_a_Scientific_Research_Tool_in_Archaeology (14.01.2013).

Koob, M. (1995) Architectura Virtualis. Konzept für das 1. Digitale Architekturmuseum, ein Wissenschaftsort für Forschung und Dokumentation der Kunst der Bautechnik im dritten kulturellen Weltgedächtnis. Fachgebiet CAD in der Architektur, Technische Universität Darmstadt, Bensheim.

Koob, M. (1995) Architectura Virtualis. Projekt Kulturerbe. Fachgebiet CAD in der Architektur, Technische Universität Darmstadt, Bensheim.

Laylock, S. D. (2008) Real-time visualisation of multiple time dependent reconstruction hypotheses for a cultural heritage site. Eurographics Digital Library. http://diglib.eg.org/ (12.11.2012).

Lercari N. (2008) Virtual Heritage in the Cloud: New Perspectives for the Virtual Museum of Bologna. Eurographics Digital Library. http://diglib.eg.org/ (23.10.2012).

Münster, S. (2011) Entstehungs- und Verwendungskontexte von 3D-CAD.Modellen in den Geschichtswissenschaften. In Meissner, K. (2011) Virtual Enterprises, Communities \& Social Networks. TUDpress, Dresden.

Pfarr, M. (2010) Documentation system for digital reconstructions. Reference to the Mausoleum of the Tang-Dynasty at Zhaoling, in Shaanxi Province, China. Proceedings of the 16th International 
Conference on Cultural Heritage and New Technologies.

http://www.stadtarchaeologie.at/?page_id=5665 (14.01.2013).

Pfarr, M. (2010) Dokumentationssystem für digitale Rekonstruktionen am Beispiel der Grabanlage Zhaoling. Technische Universität Darmstadt, tuprints. http://tuprints.ulb.tu-darmstadt.de/2302/ (14.01.2013).

Riedel, A. (2006) Modellieren, Strukturieren, Präsentieren. Von Handaufmass bis High Tech II. Informationssysteme in der historischen Bauforschung. Verlag Philipp von Zabern, Mainz.
Stead, S. (2012) Cloud Computing and Cultural Heritage Computing. Stadt Archäologie Wien. http://www.stadtarchaeologie.at/?page_id=5335 (16.12.2012).

UNESCO (2003) Charta zur Bewahrung des digitalen Kulturerbes, verabschiedet auf der 32. Generalkonferenz der UNESCO am 17. Oktober 2003.

http://www.unesco.at7user/texte/docs/charta_digital es_kulturerbe.pdf (10.05.2007). 